Relations industrielles

Industrial Relations

\title{
La situation syndicale aux Etats-Unis. Rapport d'une mission du Bureau international du travail. Genève. 1960. 202, rue Queen, Ottawa. 170 pp. \$1.25.
}

\section{Gérard Dion}

Volume 16, numéro 1, janvier 1961

URI : https://id.erudit.org/iderudit/1023985ar

DOI : https://doi.org/10.7202/1023985ar

Aller au sommaire du numéro

\section{Éditeur(s)}

Département des relations industrielles de l’Université Laval

\section{ISSN}

0034-379X (imprimé)

1703-8138 (numérique)

Découvrir la revue

Citer ce compte rendu

Dion, G. (1961). Compte rendu de [La situation syndicale aux Etats-Unis.

Rapport d'une mission du Bureau international du travail. Genève. 1960. 202, rue Queen, Ottawa. 170 pp. \$1.25.] Relations industrielles / Industrial Relations, 16(1), 131-132. https://doi.org/10.7202/1023985ar

Tous droits réservés @ Département des relations industrielles de l’Université Laval, 1961
Ce document est protégé par la loi sur le droit d'auteur. L’utilisation des services d'Érudit (y compris la reproduction) est assujettie à sa politique d'utilisation que vous pouvez consulter en ligne.

https://apropos.erudit.org/fr/usagers/politique-dutilisation/ 
alors qu'elles ne le sont que superficiellement.

Une telle conclusion découle clairement des études que l'on peut lire dans cet ouvrage, études qui ont été présentées à un symposium organisé par The Fund for the Republic, en 1958. Huit personnes bien connues dans le monde des relations du travail ont apporté leur contribution et les éditeurs, dans un chapitre spécial ont tenté de résumer les discussions.

Erich Fromm, spécialiste en psychologie, étudie le problème de la liberté dans le milieu du travail. Sumner H. Slichter, réputé pour ses travaux sur le syndicalisme, analyse la position des syndicats ouvriers dans l'économie américaine. Archibald Cox montre le rôle que peut jouer la législation dans la sauvegarde de la démocratie syndicale. David Cole et Arthur J. Goldberg insistent davantage sur la capacité que possèdent les syndicats d'arriver à la même fin grâce à leurs propres moyens. Enfin, deux expériences étrangères sont décrites: celle de l'Angleterre par Hugh A. Clegg, et celle de l'Australie par James P. McClelland.

Bien que tous les auteurs poursuivent un même but et s'entendent sur la nécessité de sauvegarder la liberté des travailleurs, la démocratie syndicale, le bien de l'ensemble de la communauté, il n'est pas facile de réconcilier des vues aussi divergentes que celles de Slichter et celles de Goldberg. Par ailleurs tous les collaborateurs insistent sur l'erreur qu'il y a à identifier la démocratie syndicale avec la démocratie politique. De plus, l'expérience démontre que l'on doit tenir compte des facteurs environnants et qu'il n'existe pas de panacée: dans un contexte différent, des moyens identiques produisent des effets contraires.

Par la nature des choses, cet ouvrage ne nous présente pas un développement logique de la question centrale qui $y$ est abordée, les travailleurs dans une société libre ». Par lui-même, cependant, il est une expression de la démocratie en donnant au lecteur la possibilité de connaître un éventail d'opinions. Il a le mérite de faire voir la difficulté du problème et la nécessité de continuer à l'approfondir, car il est évident que le mouvement ouvrier américain entre dans une nouvelle phase de son existence et doit faire face à des situations qu'il n'a pas connues auparavant.

Géraro Dion
La situation syndicale aux Etats-Unis. Rapport d'une mission du Bureau international du travail. Genève. 1960. 202, rue Queen, Ottawa. 170 pp. $\$ 1.25$.

L'Organisation internationale du travail vise à établir des normes dans le champ des relations du travail. Grâce aux conventions, les pays membres sont amenés à les accepter et à les faire respecter. Toutefois, entre la ratification d'une convention, l'établissement de mesures législatives et l'application pratique, il peut exister un décalage considérable. Pour être effectives, il ne suffit pas que les garanties du droit d'association et d'action syndicale soient consacrées par un statut, il faut qu'elles se traduisent constamment dans les faits et que leur jouissance n'en soit pas restreinte.

Plusieurs méthodes ont été successivement tentées par l'O.I.T. dans le but de remplir son rôle de surveillance d'une façon satisfaisante. D'abord l'étude des plaintes relatives à la violation des conventions. Ensuite l'étude des documents et autres sources d'information de caractère officiel. La première méthode ne peut produire que des résultats limités et superficiels. La seconde ne donne pas une vue complète de la réalité. Voilà pourquoi en 1958, le Conseil d'administration a approuvé une résolution instituant un mécanisme permettant d'obtenir des renseignements non seulement sur la situation juridique, mais encore sur la situation véritable de chacun des Etats Membres. Des missions d'études iraient faire enquête sur place dans les différents pays. Or, les deux premiers gouvernements qui ont accepté d'autoriser cet organisme extérieur à enquêter sur leur territoire sont les Etats-Unis et l'U.R.S.S. En 1959, une mission s'est rendue dans chacun de ces pays. Le Bureau international du travail vient de publier dans deux ouvrages distincts le rapport de cette mission.

La situation syndicale aux Etats-Unis est une synthèse intéressante, même pour ceux qui connaissent la vie, le développement et l'action syndicale dans ce pays. Présentée par des observateurs étrangers qui manifestent un souci d'objectivité, cette synthèse permet de saisir mieux certains aspects que l'on peut ètre porté à négliger.

Ainsi, le syndicalisme américain, pos- 
sède une histoire assez longue, mais il est une véritable force dans la nation seulement depuis une trentaine d'années. La législation a joué un rôle considérable dans l'essor ou le ralentissement de l'action syndicale. Alors que la loi existe pour affirmer l'existence et protéger la liberté d'action du syndicalisme, elle est, plus que partout ailleurs, utilisée pour brimer et entraver le fonctionnement normal des syndicats. L'application du principe de la liberté syndicale pose de nombreux problèmes. L'anti-syndicalisme est puissant. Les auteurs de l'ouvrage sont loin de partager l'opinion selon laquelle le syndicalisme aurait atteint une puissance économique et politique dangereuse pour la nation. Bien au contraire, si le syndicalisme n'est pas menacé de disparition, il rencontre des obstacles que seule une action vigilante de tous les instants pourra surmonter.

« Rares sont les sociétés démocratiques, lit-on dans le rapport, où les circonstances ont été aussi peu favorables au syndicalisme qu'aux Etats-Unis. Aussi l'histoire du syndicalisme américain estelle caractérisée par une suite de luttes, souvent violentes, bien que séparées par des périodes d'accalmie et de progrès dans les relations professionnelles. \ L'opinion publique n'est pas encore gagnée au syndicalisme. Dans un langage qui ne manque pas de diplomatie, les auteurs du rapport disent: « Même si l'on ne conteste pas la place qu'a acquise le syndicalisme, il est encore trop tốt pour affirmer que l'opinion publique dans son ensemble est convaincue que celui-ci constitue une composante souhaitable et nécessaire de la vie américaine $\$$.

Les employeurs disposent de puissants moyens de propagande et l'on se demande parfoís sí, sous le couvert d'une légitime divergence de vues et d'intérêts, ne se cache pas une opposition à l'existence même du syndicalisme. « Refuser catégoriquement, lors d'une négociation, telle revendication précise des syndicats n'exclut pas la reconnaissance des dits syndicats. Mais quand la propagande est menée par voie d'affiches, de « conférences forcées 》dans l'entreprise, de lettres adressées à domicile aux salariés et à leurs femmes, ou de campagnes de presse locales visant à dissuader les travailleurs de constituer un syndicat ou d'y adhérer, on peut supposer qu'il ne s'agit plus de s'opposer aux revendications syndicale, mais au syndicalisme luimême. La même supposition s'impose à l'esprit quand on constate que certains employeurs s'efforcent d'empêcher un syndicat de prendre pied dans une entreprise en faisant savoir aux travailleurs que l'existence d'un tel syndicat pourrait entraîner la fermeture de l'entreprise, même si de tels propos ne constituent pas une menace, mais simplement * une prédiction ou une prophétie ». (p. 167)

Les syndicats peuvent-ils compter sur les partis politiques pour obtenir une législation leur permettant une liberté d'action garantie? Les partis réflètent l'opinion publique, et l'on retrouve dans leur sein des éléments très conservateurs. « La coalition qui a assuré le triomphe du projet Landrum-Griffin, soutient le rapport, incarne les tendances que les syndicats ont les meilleures raisons de craindre, à savoir les éléments les plus conservateurs des deux partis. Les syndicats reçoivent ainsi la démonstration de la fragilité de leur puissance, de leur baisse de prestige auprès de l'opinion publique et du Congrès et de l'efficacité des moyens que les employeurs peuvent mettre en oeuvre pour contenir la poussée syndicale 》. (pp. 139-140)

Pour surprenant que cela puisse paraître à quelques-uns, les observateurs impartiaux de l'O.I.T. en vont jusqu'à écrire: «Il est à peine exagéré de dire que les syndicats n'ont été pleinement admis par l'opinion publique qu'en temps de crise nationale, c'est-à-dire au cours des deux guerres mondiales et lors de la grande crise économique. Tout se passe comme si les syndicats des Etats-Unis fonctionnaient dans un système social qu'ils acceptent, mais qui ne les accepte pas encore complètement ». (pp. 28-29) Un tel jugement ne plaira pas à tous, mais il vaut la peine qu'on s'y arrête si l'on veut continuer à présenter le système économique et politique des EtatsUnis comme un idéal aux autres nations.

\section{Géraro Dion}

La situation syndicale en U.R.S.S.. Rapport d'une mission du Bureau international du travail, Genève, 1960 . 202, rue Queen, Ottawa, 152 pp. $\$ 1.25$.

Nous avons lu La situation syndicale en U.R.S.S. immédiatement après l'ouvrage consacré à la même question aux Etats-Unis. Nons ne pouvons pas nous empêcher d'être frappé par l'extrême 